\title{
Characteristics of flow fields in the gas-liquid mini-bubble columns with PIV measurements
}

\author{
Chen $\mathrm{Li}^{1}$, Yongli Ma ${ }^{1}$, Ming-yan $\mathrm{Liu}^{1}$, and Kaiyue Wang ${ }^{1}$ \\ ${ }^{1}$ Tianjin University
}

February 2, 2022

\begin{abstract}
As a new type of gas-liquid microreactors, the gas-liquid mini-bubble column have potential applications. However, few studies on the flow fields in the mini-bubble column can be found at present. In this work, Particle Image Velocimetry (PIV) was first used to visually study the velocity fields, vorticity fields and bubble dynamics in the gas-liquid mini-bubble columns with column inner diameters of 1 to $3 \mathrm{~mm}$ and mini-bubble diameters ranged from 0.7 to $1.3 \mathrm{~mm}$. It is found that with the increase of superficial liquid velocity, bubbles rose from almost straight line to Z-shaped or S-shaped trajectory, and the bubble trajectory changed from 1-D to 3-D; when the bubble velocity changed, the bubble size and gas holdup decreased; bubble terminal velocity was controlled by bubble buoyancy and flow resistance, and increased slightly with bubble coalescence. These findings may provide basic reference for the design and scale-up of such mini-bubble column reactor.
\end{abstract}

\section{Hosted file}

20220124-Manuscript-Final-Sent.docx available at https://authorea.com/users/458443/articles/ 554993-characteristics-of-flow-fields-in-the-gas-liquid-mini-bubble-columns-with-pivmeasurements

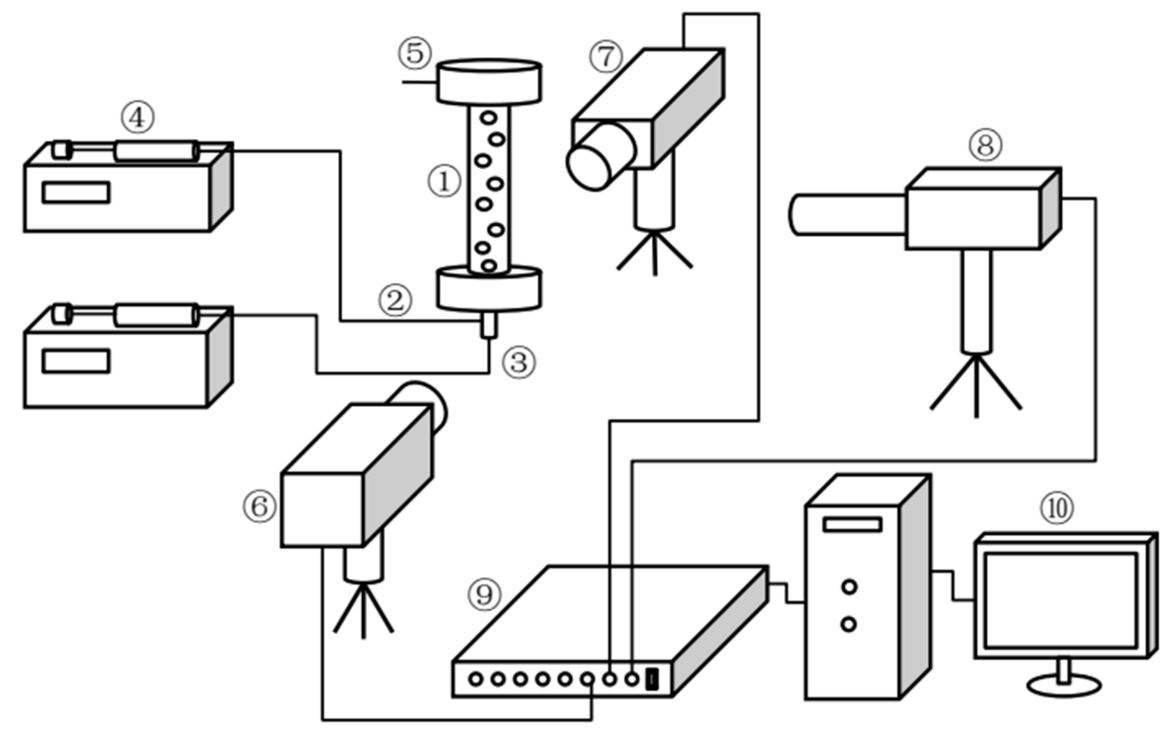



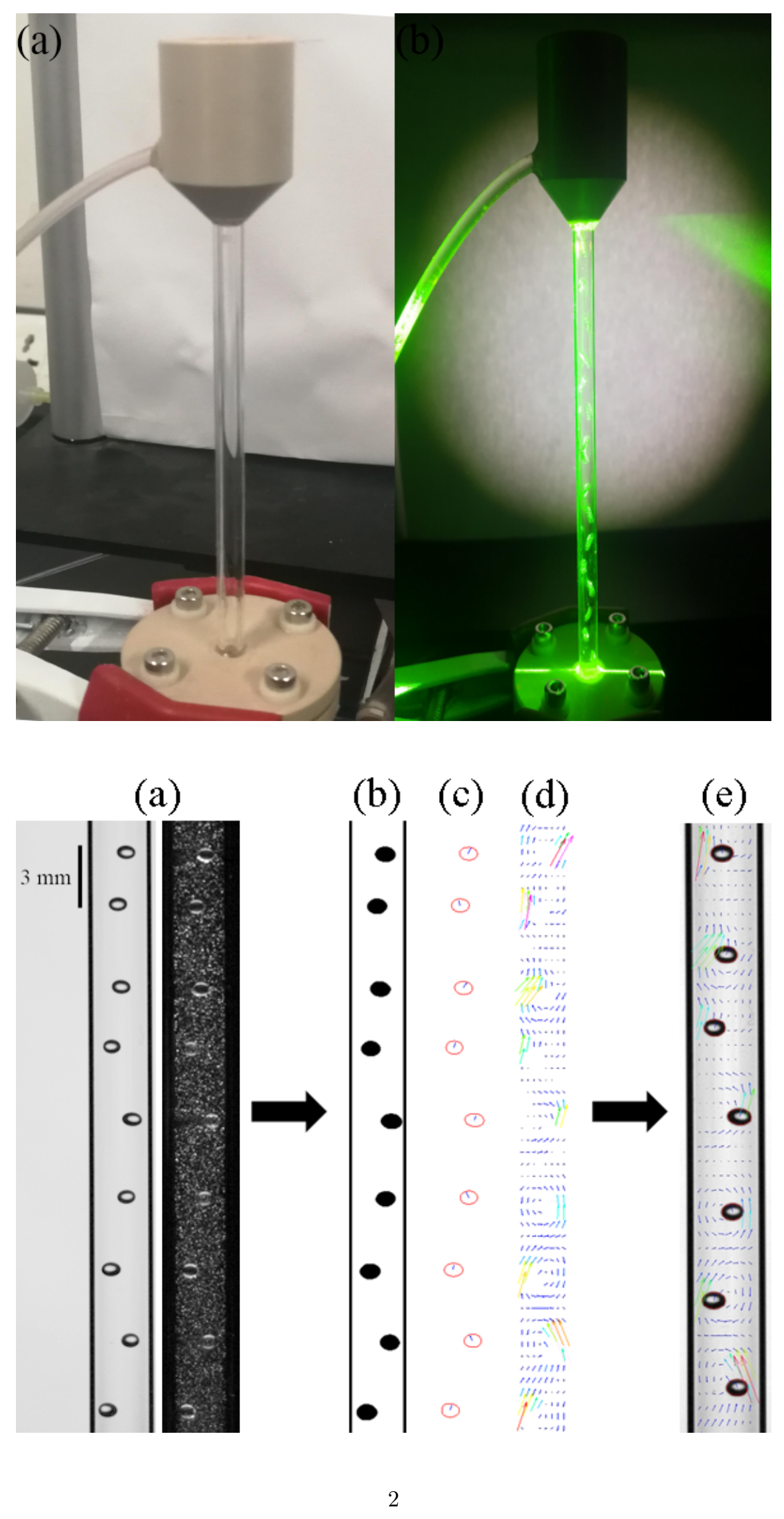


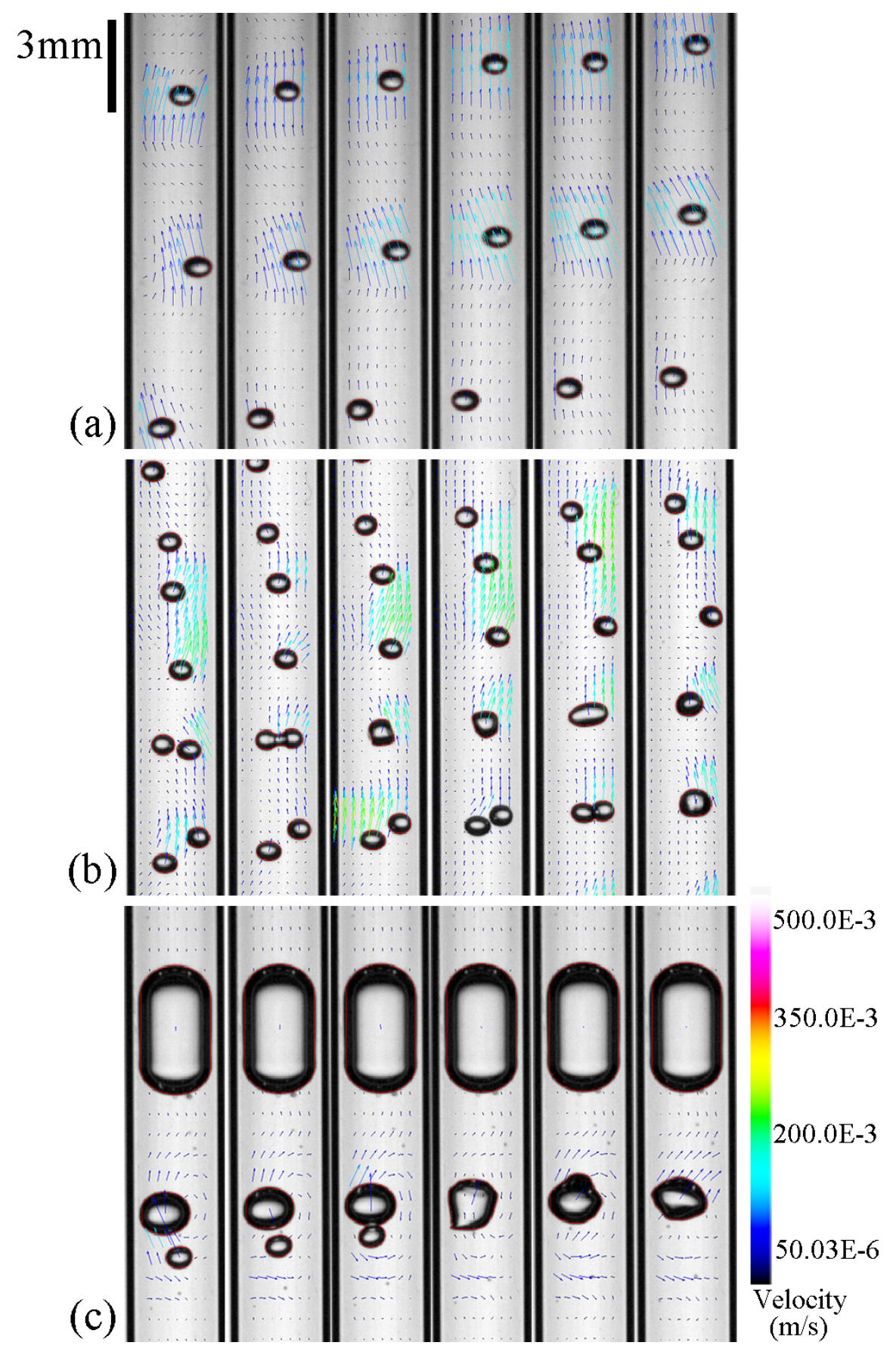



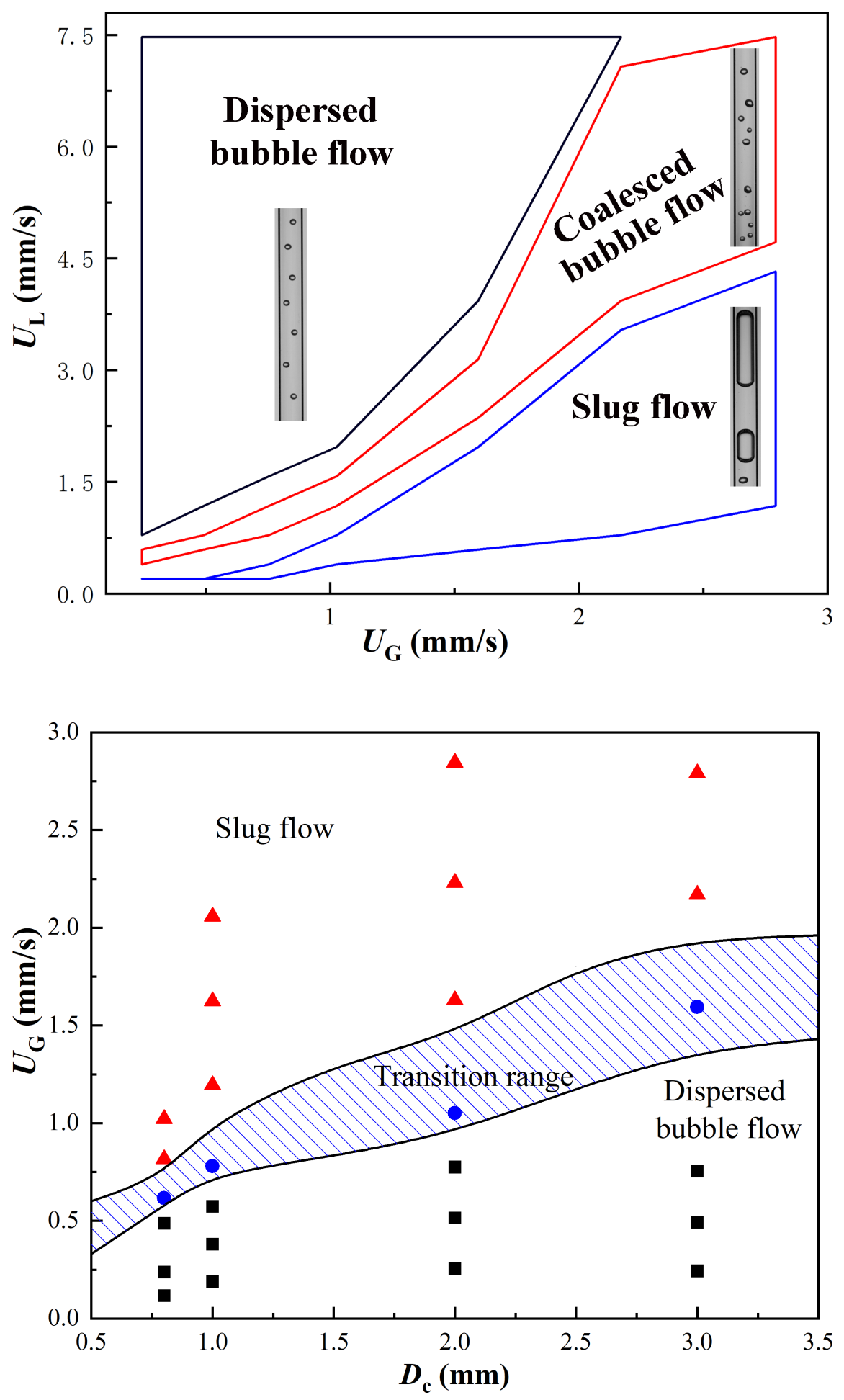


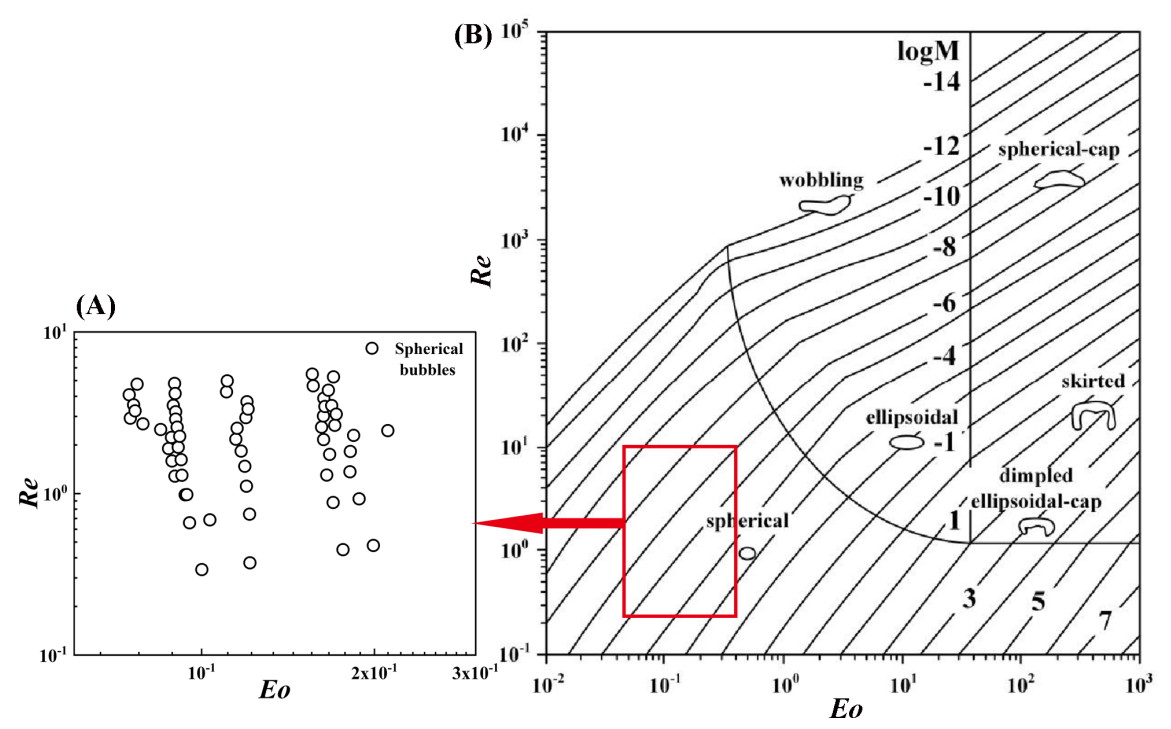




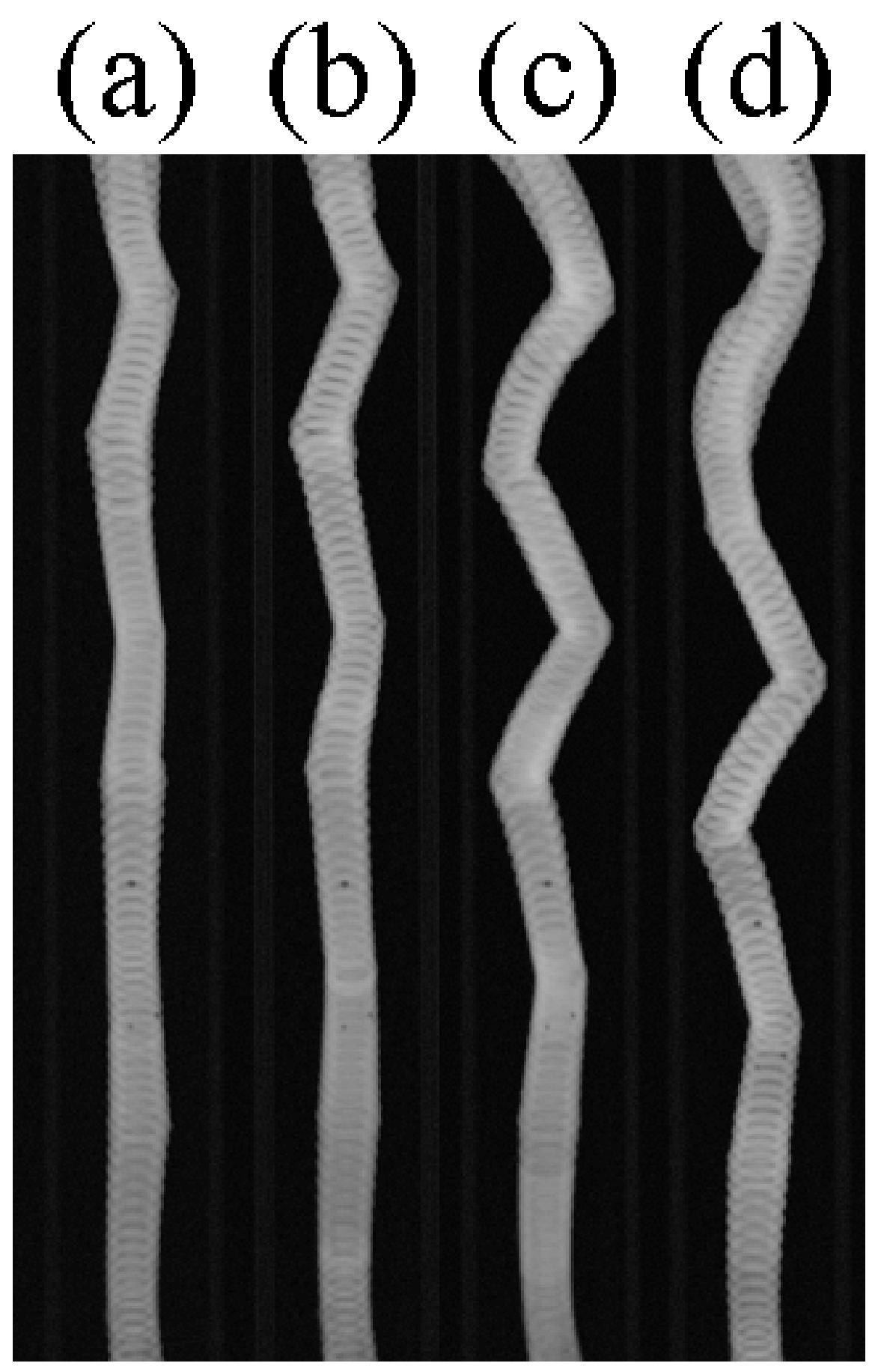




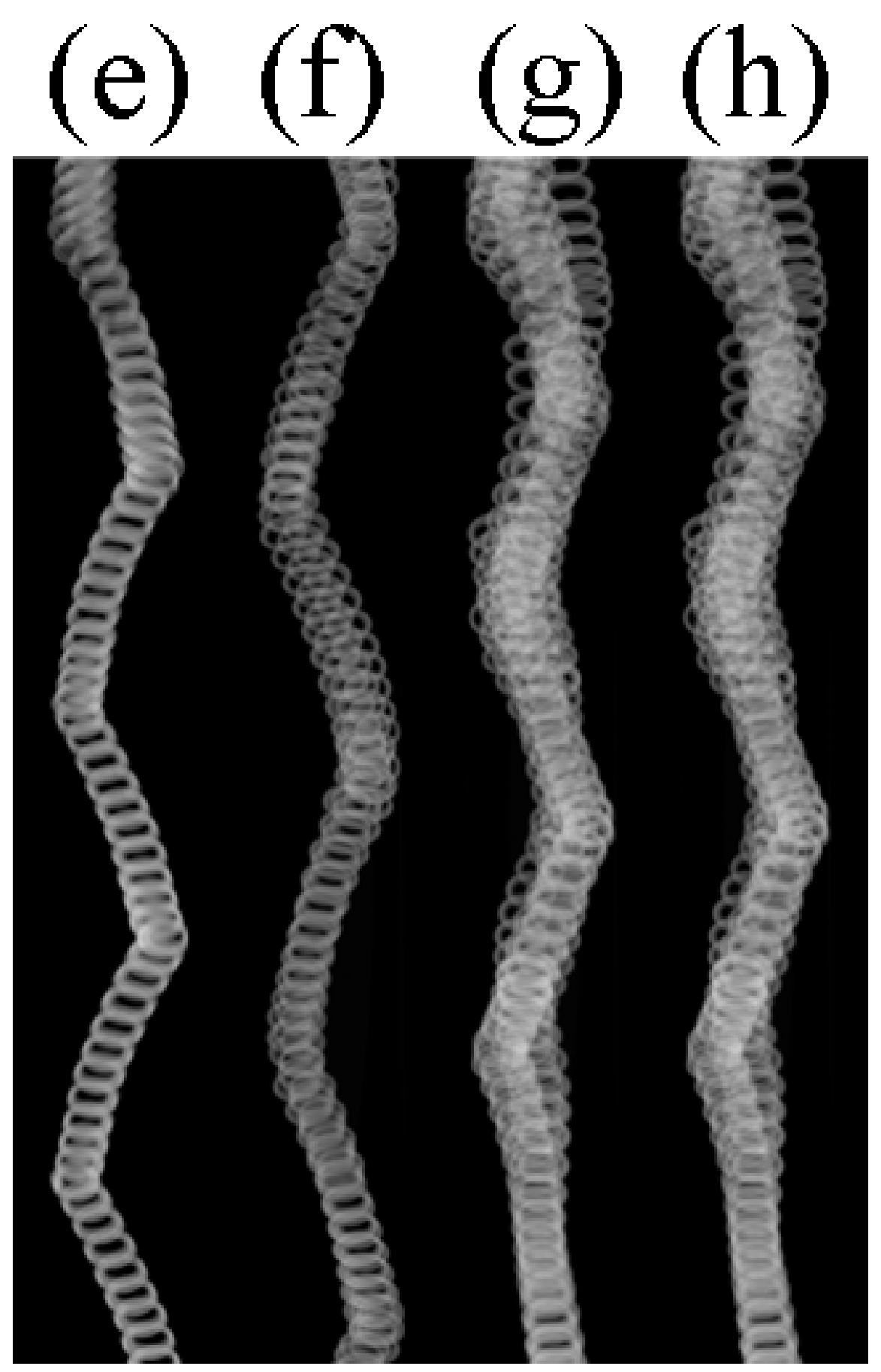




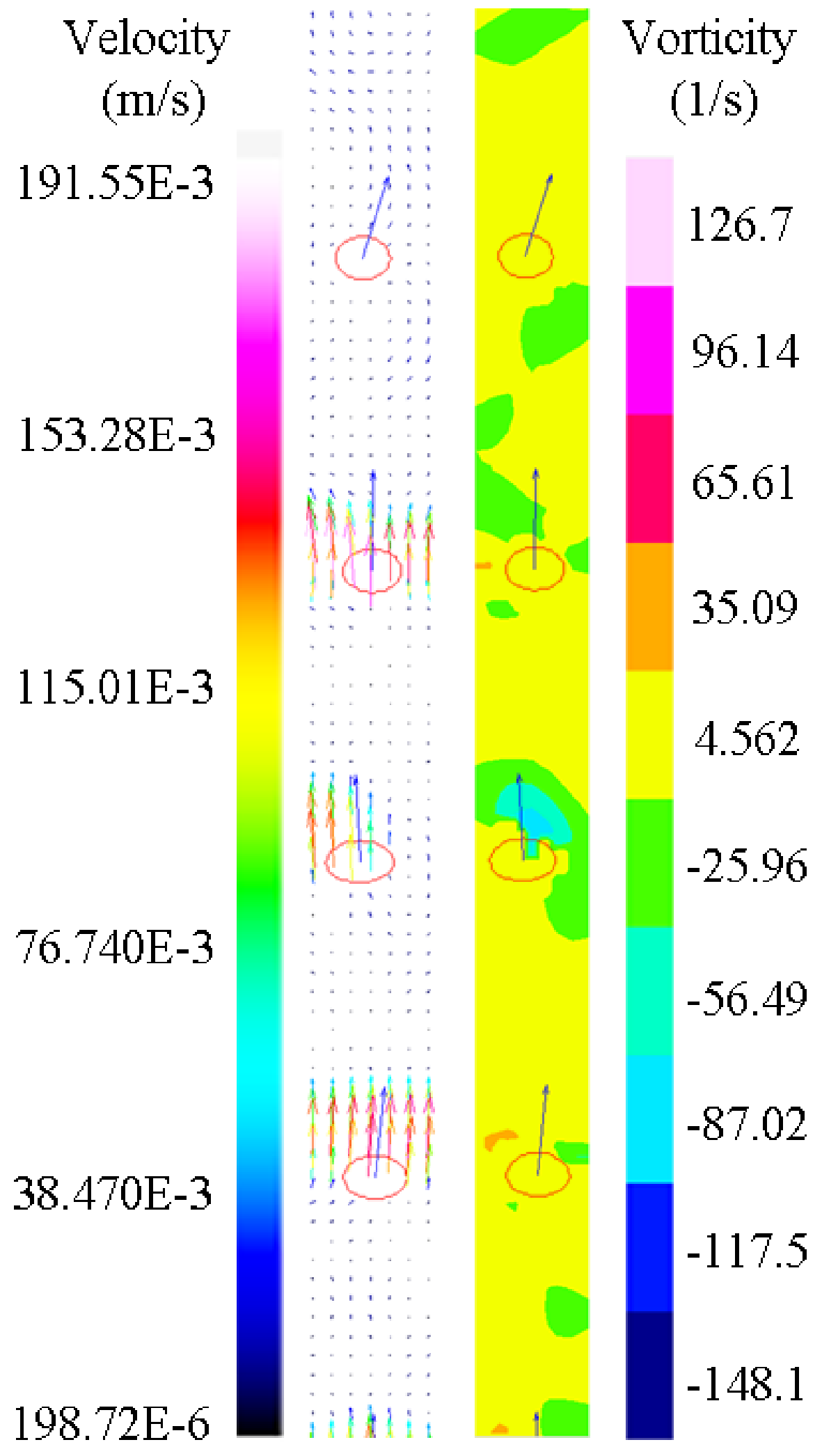




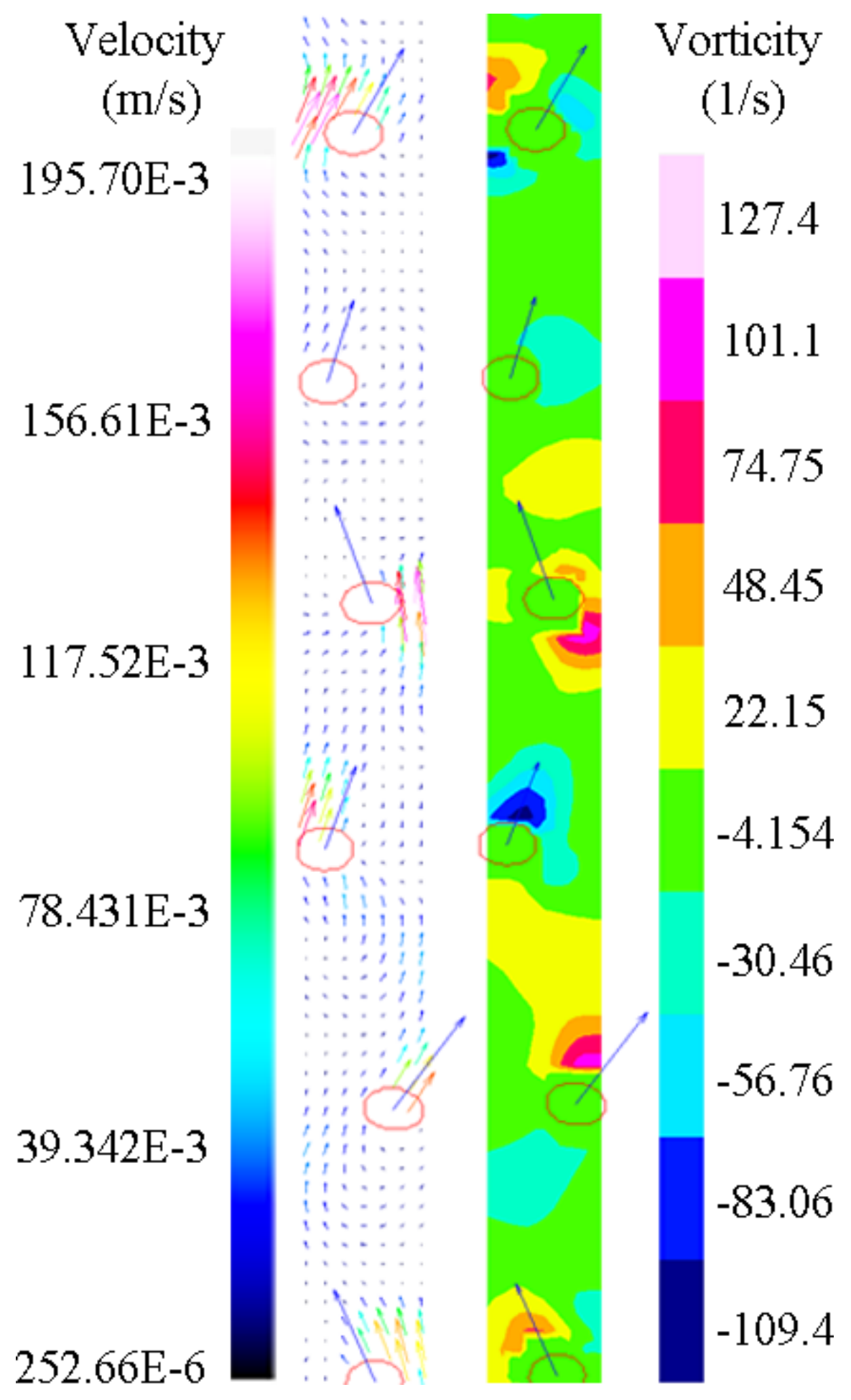




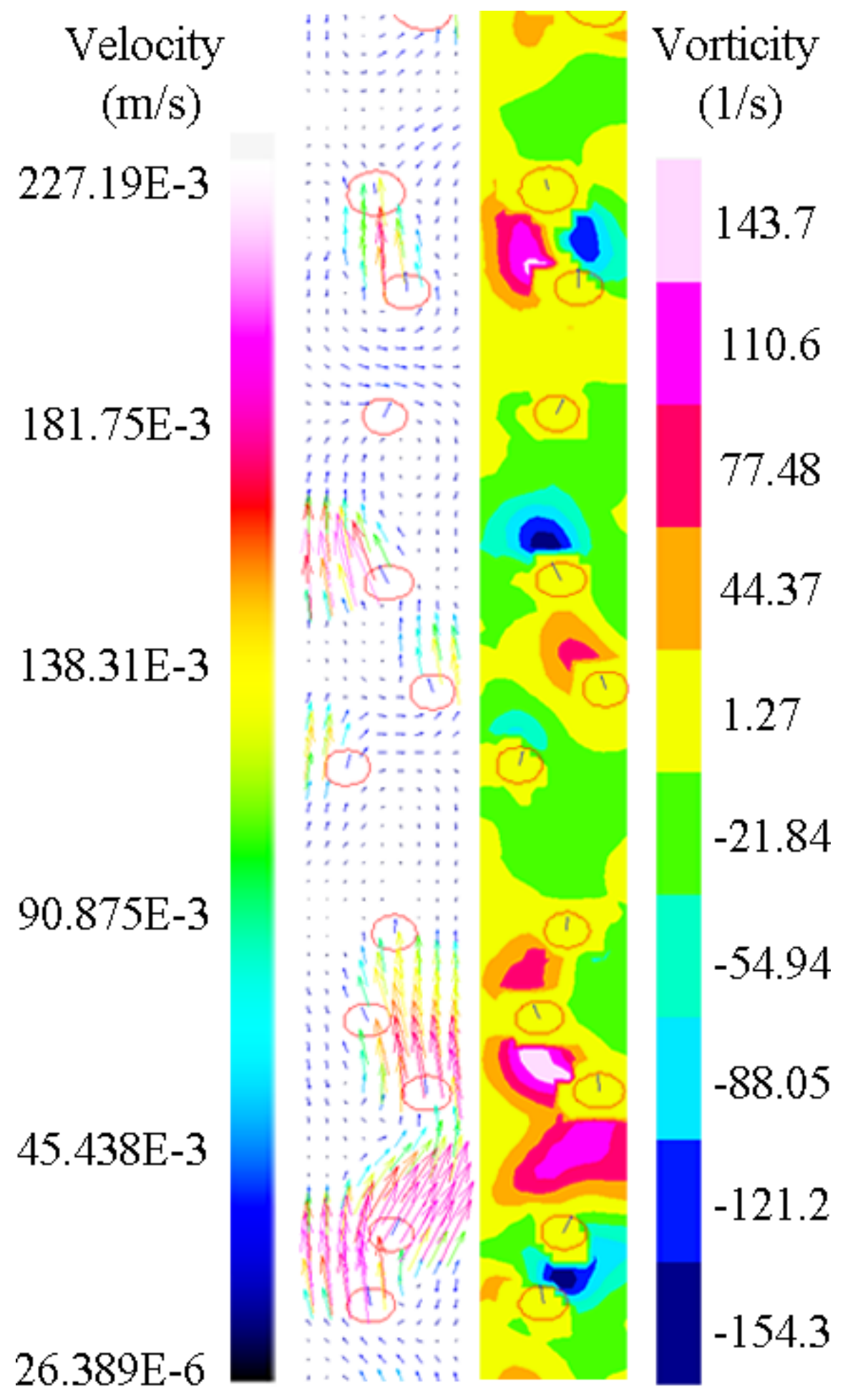



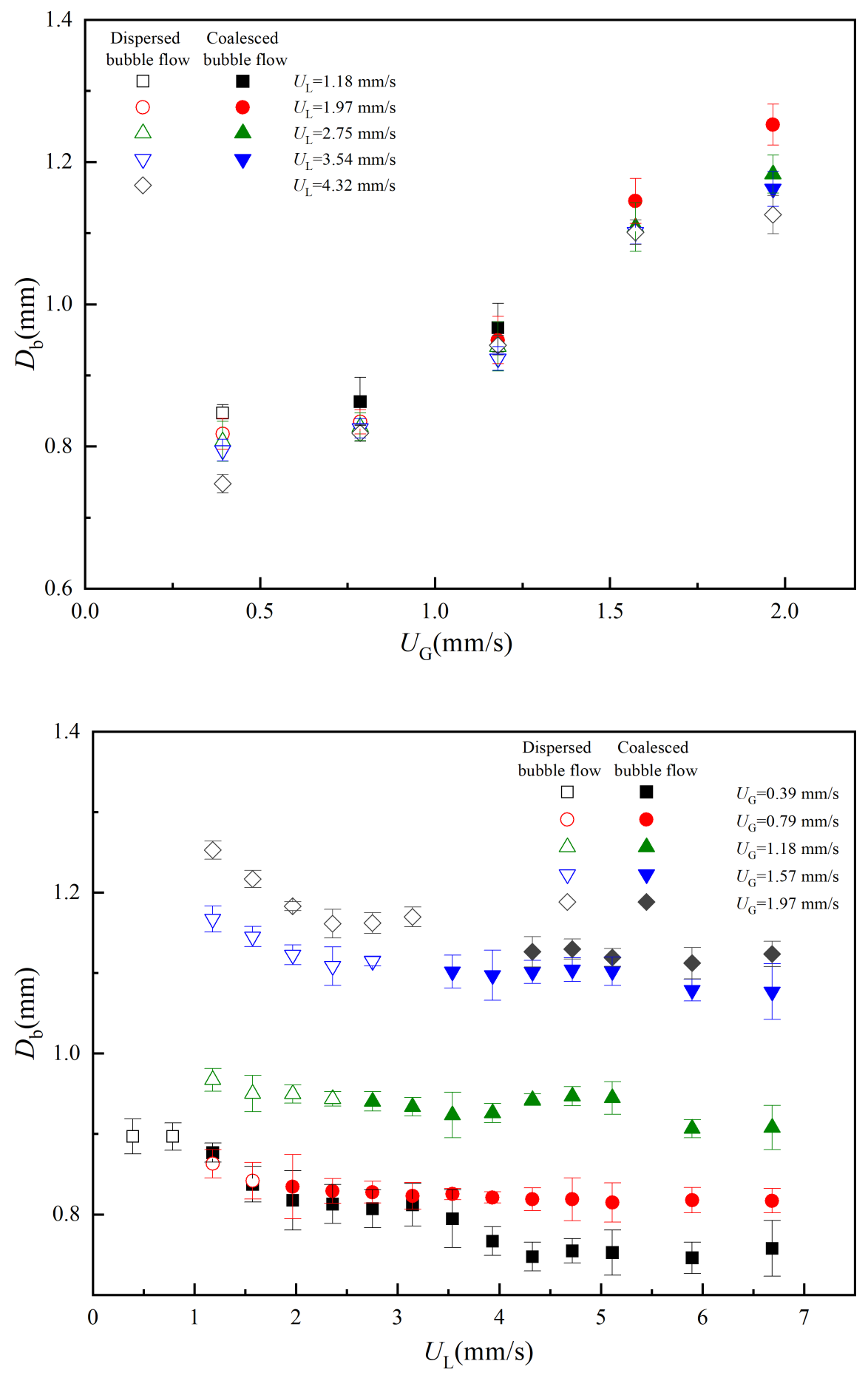
(a)

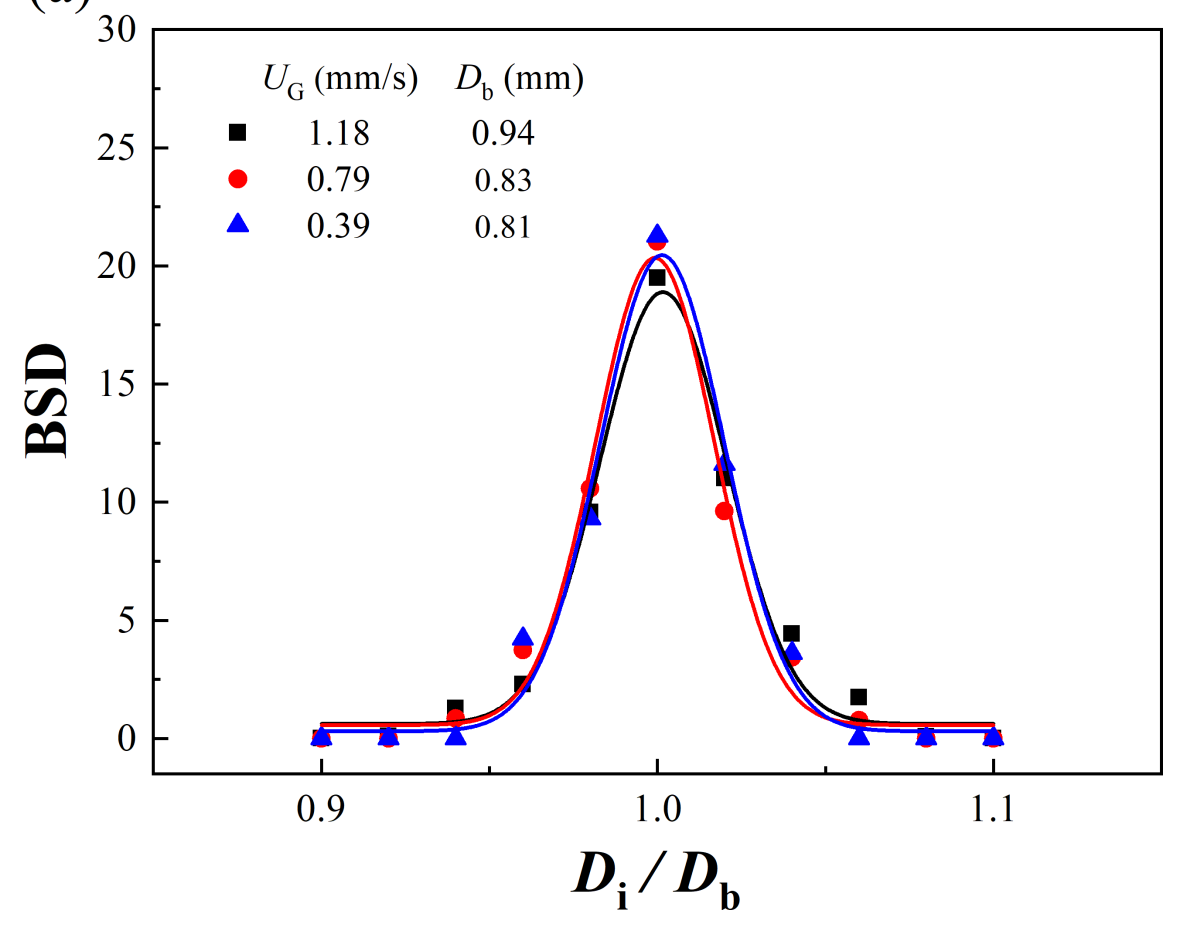

(b)

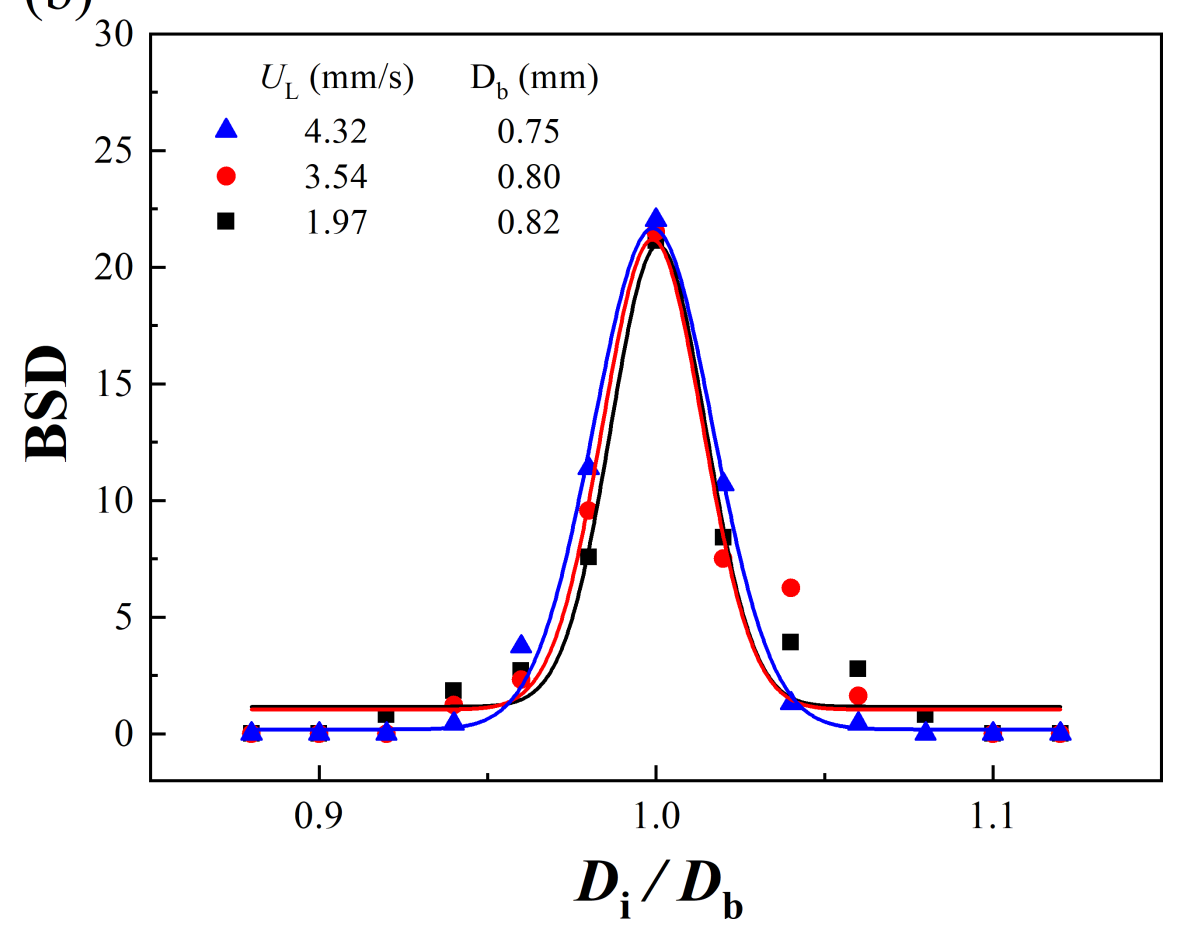



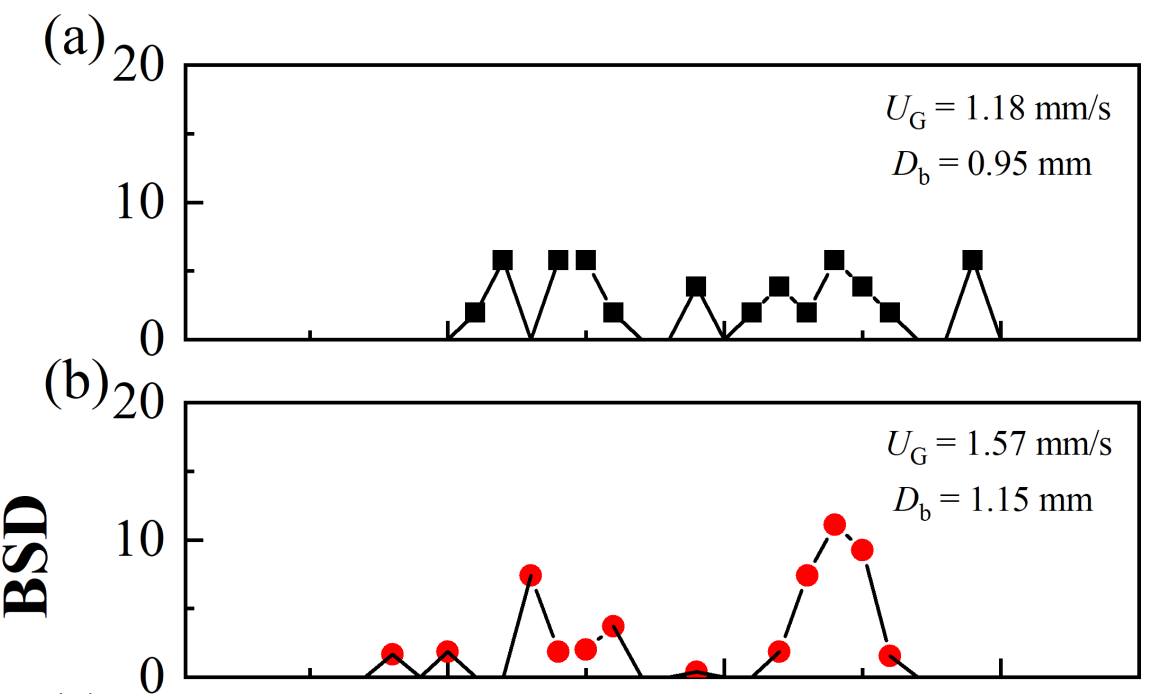

(c)

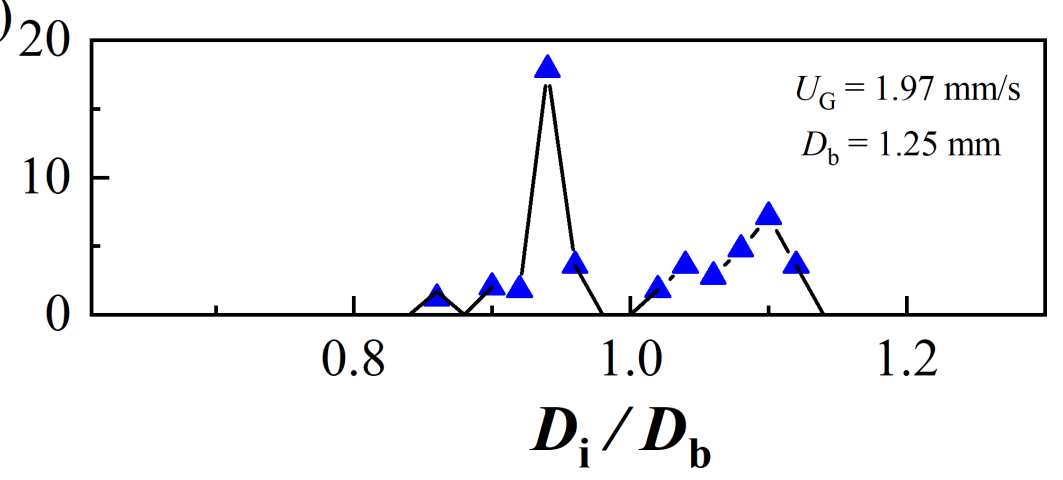



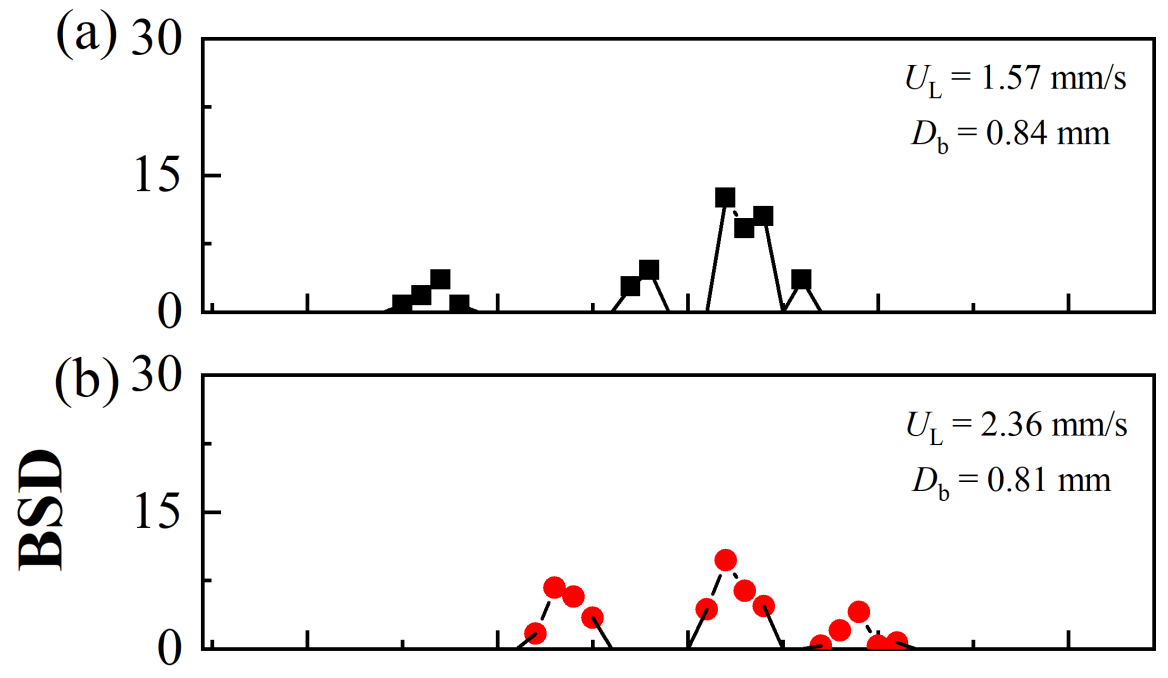

(c) 30
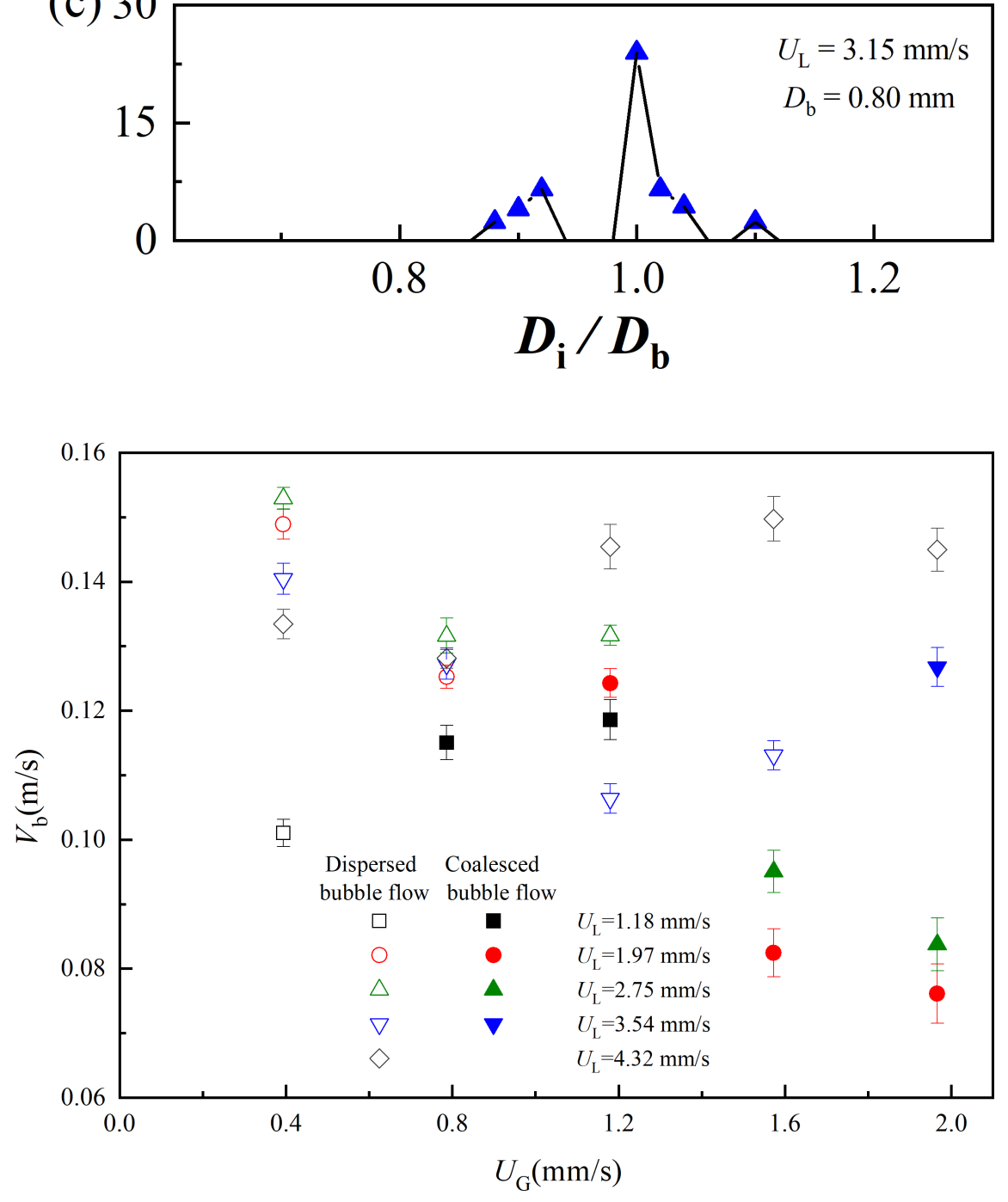

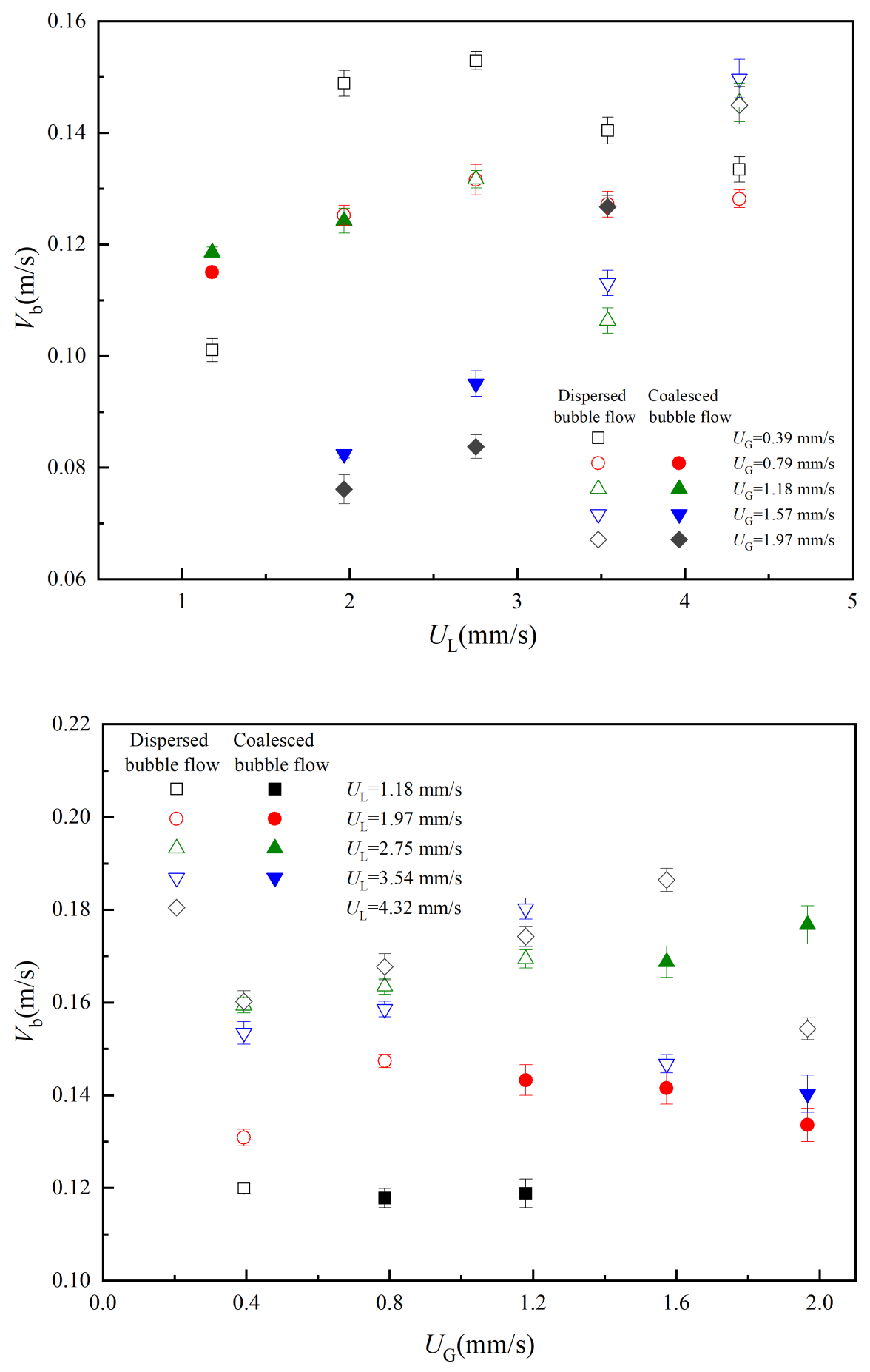

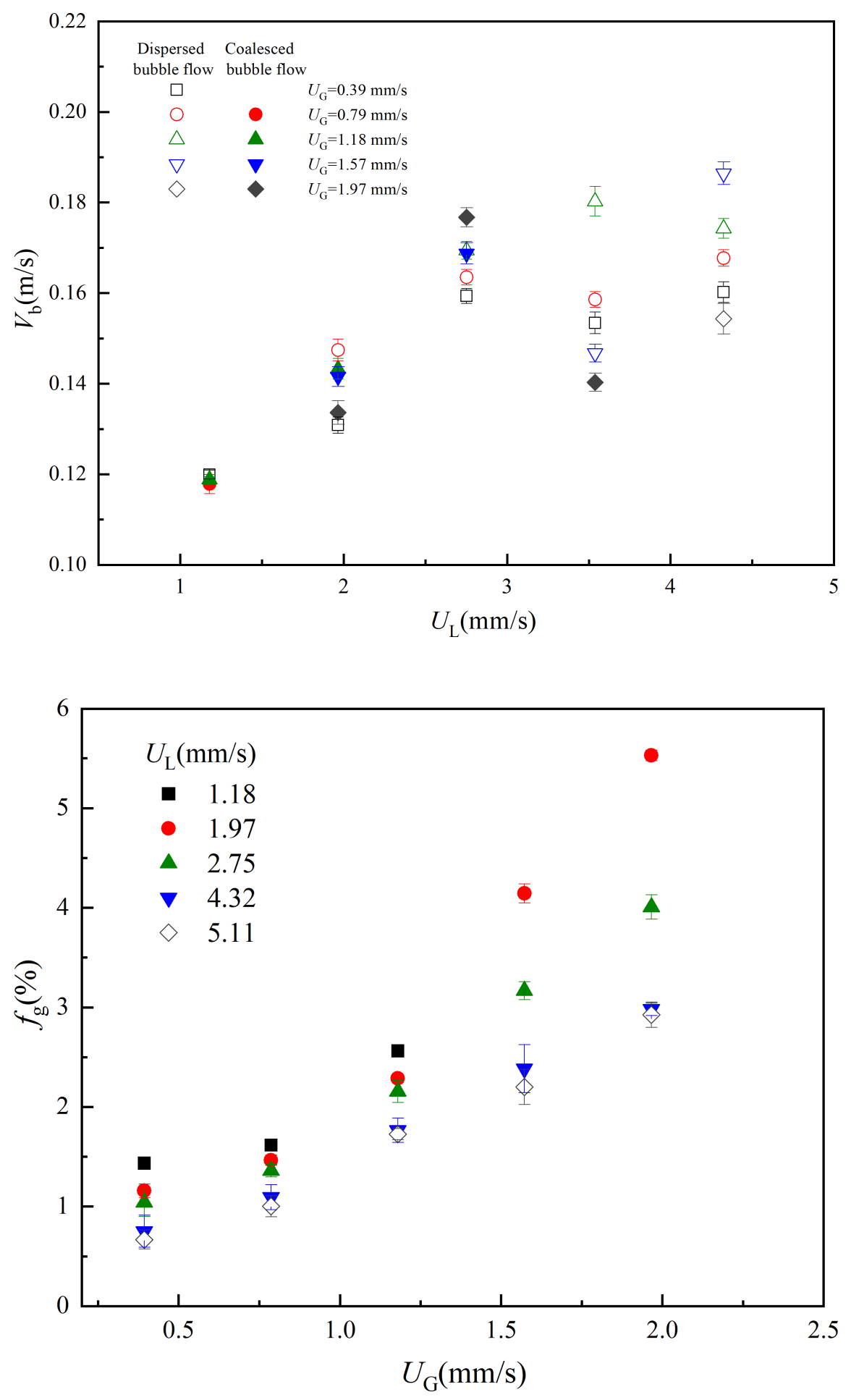


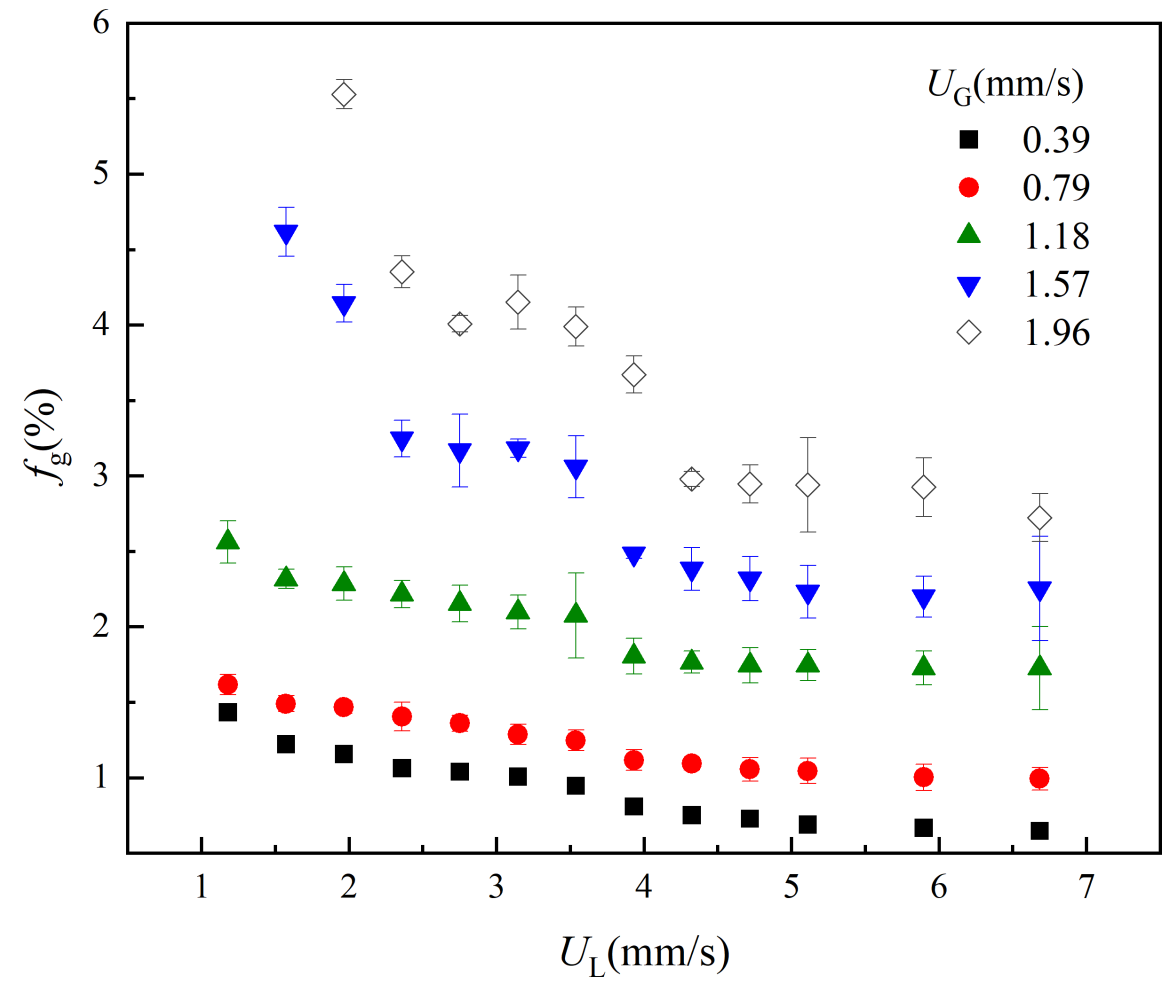

\section{Hosted file}

Table1.docx available at https://authorea.com/users/458443/articles/554993-characteristicsof-flow-fields-in-the-gas-liquid-mini-bubble-columns-with-piv-measurements

\section{Hosted file}

Table2.docx available at https://authorea.com/users/458443/articles/554993-characteristicsof-flow-fields-in-the-gas-liquid-mini-bubble-columns-with-piv-measurements

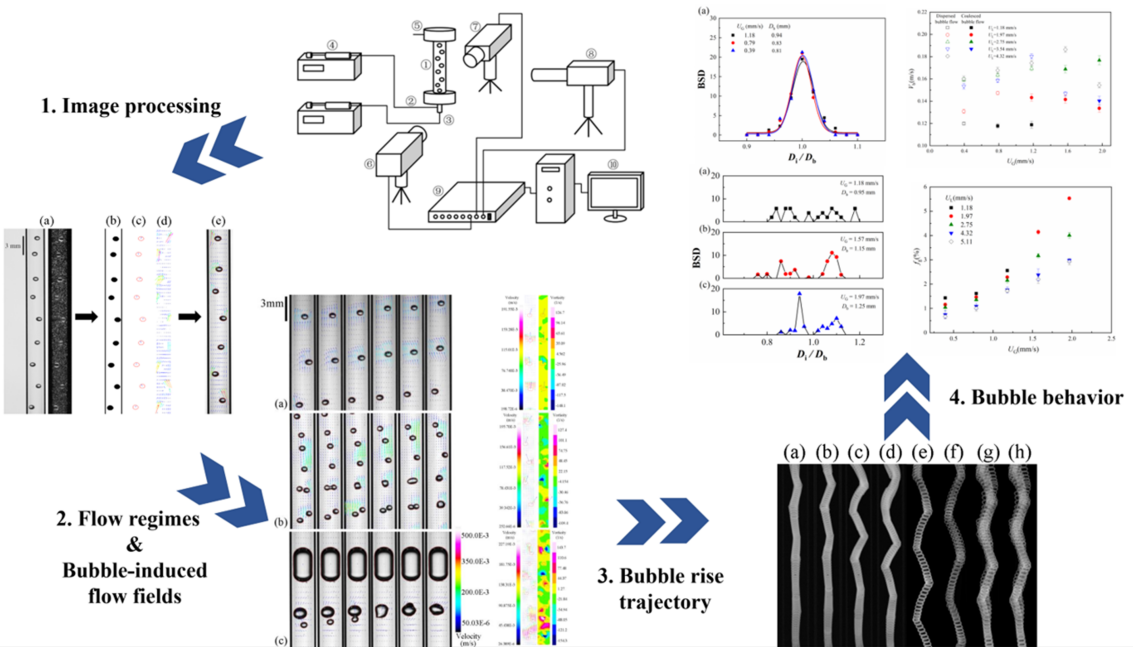

